\title{
Aderência à Madeira de Polímeros Reforçados com Fibras de Carbono sob Temperaturas Elevadas
}

\author{
Lucas Rubini, Poliana Dias de Moraes \\ Departamento de Engenharia Civil, Universidade Federal de Santa Catarina - UFSC
}

\begin{abstract}
RESUMO
O objetivo deste trabalho é avaliar a aderência de reforços compósitos de fibra de carbono e resina epoxídica a elementos de madeira entre 20 e $80^{\circ} \mathrm{C}$. Para tanto, utilizou-se madeira da espécie Pinus taeda, com massa específica aparente média de $554 \mathrm{~kg} / \mathrm{m}^{3}$ e teor de umidade à temperatura ambiente de $12,0 \%$. O reforço utilizado foi manta de fibra de carbono com adesivo à base de epóxi. A amostra foi constituída por 40 corpos de prova, os quais foram ensaiados às temperaturas de $20,40,60$ e $80^{\circ} \mathrm{C}$. Chegou-se à conclusão de que a resistência do reforço diminui com o aumento da temperatura, verificando-se a ruptura pela linha de cola em todos os níveis de temperatura de ensaio. Ainda, pela análise de resistência relativa, verificou-se uma redução de 38,3 e $65,3 \%$ na resistência a 60 e a $80^{\circ} \mathrm{C}$, respectivamente, em relação àquela obtida a $20^{\circ} \mathrm{C}$.
\end{abstract}

Palavras-chave: adesivos poliméricos, reforço estrutural, temperatura.

\section{Adhesion to Wood of Carbon Fiber Reinforced Polymers at High Temperatures}

\begin{abstract}
The purpose of this study was to assess the adherence of carbon fiber composite reinforcements and epoxy resin to wood elements between 20 and $80{ }^{\circ} \mathrm{C}$. To this end, wood samples from Pinus taeda were used, with an average bulk density of $554 \mathrm{~kg} / \mathrm{m}^{3}$ and moisture content at room temperature of $12.0 \%$. Carbon fiber cloth with epoxy adhesive was used as reinforcement. The sample consisted of 40 specimens, which were tested at the temperatures of $20,40,60$ and $80^{\circ} \mathrm{C}$. It was possible to conclude that the reinforcement strength decreases with the increase of temperature, as verified by the rupture at the glue line in all test temperature levels. Still, the analysis of relative strength showed a reduction of 38.3 and $65.3 \%$ in the resistance at 60 and $80^{\circ} \mathrm{C}$, respectively, compared to that obtained at $20^{\circ} \mathrm{C}$.
\end{abstract}

Keywords: polymeric adhesives, structural reinforcement, temperature.

\section{INTRODUÇÃO}

A madeira, um material de grande disponibilidade e acesso, possui elevada resistência mecânica à tração e à compressão, além de proporcionar bom isolamento térmico, elétrico e acústico. Devido à sua facilidade de manuseio e processamento, tornou-se uma das primeiras e principais matérias-primas a serem empregadas pelo homem. Sua utilização abrange as mais diversas aplicações, como a Construção Civil e a Arquitetura, a indústria papeleira e de energia, a marcenaria, entre outros. Apesar disso, alguns 
aspectos devem ser levados em conta quanto à sua utilização, particularmente no que diz respeito à atuação da madeira em diferentes temperaturas.

No intuito de aumentar a resistência da madeira, novas técnicas têm sido estudadas, entre elas o uso de materiais de reforço. Em elementos estruturais em madeira, o reforço estrutural visa atenuar a variabilidade das propriedades mecânicas decorrentes da anisotropia desse material. Uma das técnicas de aplicação de reforços é o uso de adesivos poliméricos. Os polímeros reforçados com fibras (PRF, ou FRP - Fibre Reinforced Polymer), que, geralmente, utilizam fibra de vidro (PRFV), de carbono (PRFC) ou de aramida (PRFA), apresentam valores de resistência e rigidez muito elevados em relação à massa específica própria. Ainda, por serem materiais não metálicos, possuem boa resistência à corrosão, são de fácil manejo e oferecem espessura mínima, o que evita a alteração das dimensões dos elementos estruturais, conservando e mantendo os critérios de projeto e funcionalidade original da estrutura.

Entretanto, a escolha dos materiais de reforço depende da localização do elemento estrutural na edificação, da facilidade de acesso ao local, da resistência ao fogo requerida pela estrutura, do resultado estético pretendido e dos custos envolvidos na recuperação ou reforço do elemento. Alguns exemplos do uso dos FRP podem ser encontrados na reabilitação de pontes de madeira, como a Ponte em arco de Westchester, nos Estados Unidos (Garcez et al., 2004); na preservação do patrimônio histórico e na reabilitação de habitações, como a Basílica de São Francisco de Assis, na Itália (Motavalli \& Czaderski, 2007); no reforço de estruturas contra a ação sísmica no Japão, como a ponte de Okinawa Road Park (Bakis et al., 2002; Nishizaki et al., 2006).

Existe uma grande variedade de adesivos que podem ser utilizados em combinação com as fibras, tais como resinas poliéster, vinílicas e epoxídicas (Fiorelli \& Dias, 2002). Os adesivos epoxídicos são amplamente utilizados devido à sua boa resistência às ações do ambiente e à capacidade de ligar-se a uma vasta gama de superfícies, incluindo madeira, metais, plásticos, cerâmica e concreto (FRIHART, 2005). A escolha pelo tipo ideal de adesivo depende das condições ambientais e estruturais da aplicação do compósito, porém o adesivo mais utilizado atualmente é a resina epoxídica.

Inúmeras pesquisas vêm sendo efetuadas sobre madeira reforçada, tais como questões relacionadas a sobrecargas, ao efeito da umidade e da durabilidade e, mais recentemente, questões ligadas ao comportamento ao fogo e à temperatura. No entanto, em relação a esta última, o conhecimento é ainda insipiente e ainda há um longo caminho a se percorrer.

Dessa forma, este trabalho tem como objetivo avaliar a aderência de reforços compósitos de fibra de carbono e da resina epoxídica a elementos de madeira sob a ação da temperatura, buscando-se adequar uma metodologia para determinação da aderência entre elementos de madeira e reforços compósitos de fibra de carbono e resina epoxídica para temperaturas elevadas.

\section{MATERIAIS E MÉTODOS}

\subsection{Materiais}

A madeira utilizada neste trabalho foi da espécie Pinus taeda, com massa específica aparente média de $554 \mathrm{~kg} / \mathrm{m}^{3}$ e teor de umidade à temperatura ambiente de $12,0 \%$, proveniente de florestas plantadas do Estado de Santa Catarina, Brasil.

O reforço utilizado foi manta fibra de carbono (PRFC), com a designação comercial de SikaWrap 230C (Figura 1a). O adesivo que interligou os materiais foi resina à base de epóxi, designada comercialmente por MC-DUR 1209 (Figura1b), sendo essa constituída por dois componentes: base e endurecedor (catalisador), os quais são misturados

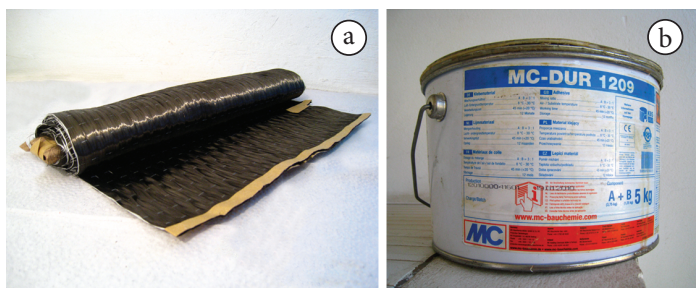

Figura 1. Fibra de carbono SikaWrap ${ }^{\circledR}-230 \mathrm{C}$ (a); Epoxi MC-DUR 1209 (b).

Figure 1. SikaWrap ${ }^{\circ}-230 \mathrm{C}$ carbon fiber (a); MCDUR 1209 epoxy resin (b). 
numa proporção de 3:1, conforme indicação do fabricante (MC-Bauchemie, 2011).

Os elementos de madeira usados para a fabricação dos corpos de prova apresentavam dimensões de $2,50 \mathrm{~m}$ de comprimento e seção transversal de $10,00 \times 5,00 \mathrm{~cm}$, aproximadamente, e foram selecionados previamente de um lote heterogêneo por meio de uma classificação visual segundo o anexo G do Projeto ce-02:126.10 (ABNT, 2003), a fim de garantir a isenção de defeitos, como nós, rachaduras e fendas, e empenamentos nos corpos de prova.

\subsection{Amostra e corpos de prova}

A amostra estudada era constituída por 40 corpos de prova, os quais foram divididos em quatro grupos de massas específicas aparentes estatisticamente homogêneas (Montgomery \& Runger, 2003), a serem ensaiados às temperaturas de $20,40,60$ e $80^{\circ} \mathrm{C}$.

A partir dos resultados de Balseiro (2007), determinaram-se as dimensões do corpo de prova para o estudo da resistência do reforço. A fixação desse à máquina de ensaio foi feita por meio de pinos, para que não houvesse esmagamento perpendicular às fibras nas extremidades. Neste estudo, o comprimento de aderência foi de $3,00 \mathrm{~cm}$ (Figura 2).

\subsection{Pré-aquecimento e ensaios mecânicos}

Os corpos de prova foram pré-aquecidos por 120 minutos em forno elétrico na temperatura definida para o ensaio, a qual era mantida pela câmara térmica. Os ensaios mecânicos foram realizados na máquina universal de ensaios Kratos, modelo K 20000MP, interface ICKL1-USB (Figura 3), às temperaturas de 20, 40, 60 e $80^{\circ} \mathrm{C}$, com célula de carga de $50 \mathrm{kN}$, até que ocorresse a ruptura dos corpos de prova. A aplicação da carga no corpo de prova foi realizada pelo deslocamento da travessa com velocidade de $2 \mathrm{~mm} / \mathrm{min}$.

\subsection{Determinação do teor de umidade}

A determinação do teor de umidade foi realizada utilizando-se os próprios corpos de prova ensaiados nos testes mecânicos, porém seguindo o critério de temperatura e os procedimentos de pesagem definidos pela norma NBR 7190 (ABNT, 1997), Anexo B. Depois do ensaio mecânico, os corpos de prova foram pesados para a determinação da massa no final do ensaio e, em seguida, submetidos à secagem a uma temperatura máxima de $103 \pm 2{ }^{\circ} \mathrm{C}$, até a ocorrência de uma variação, entre duas medidas consecutivas, menor ou igual a $0,5 \%$ da última massa medida, sendo esta considerada a massa seca. O teor
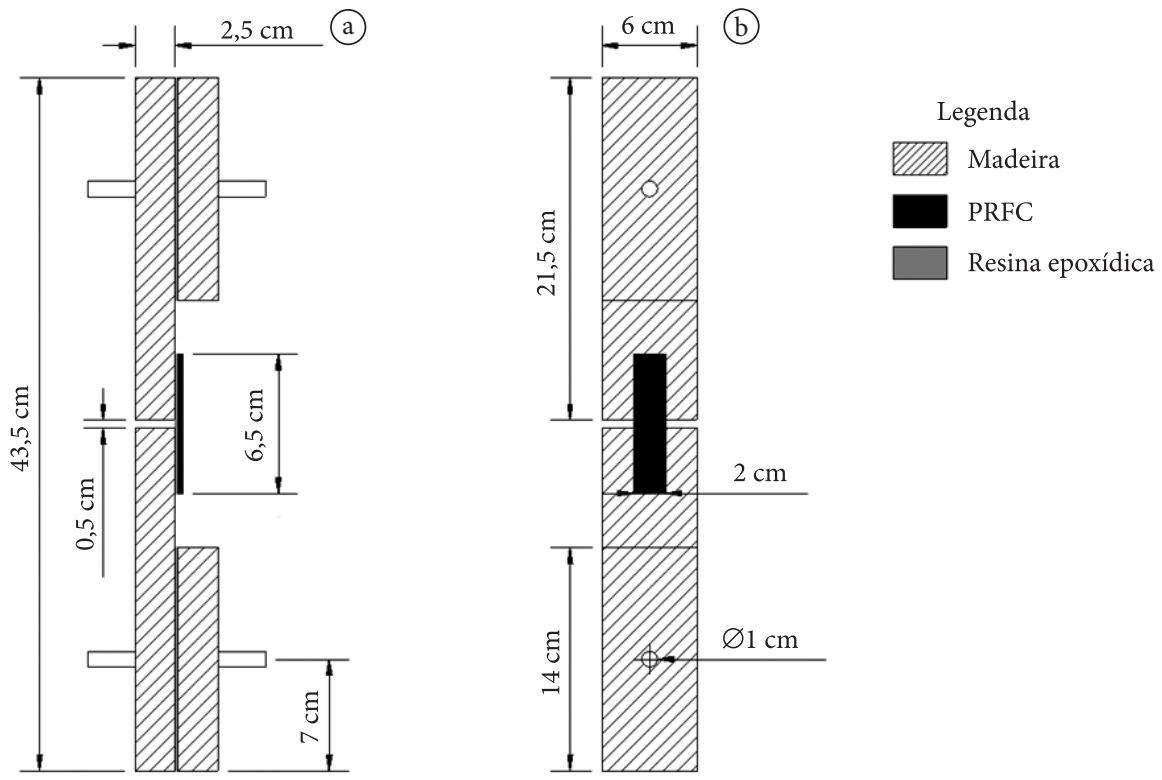

Figura 2. Corpo de prova com reforço: vista lateral (a); vista frontal (b).

Figure 2. Specimen with reinforcement: side view (a); front view (b). 
de umidade foi então calculado pela razão entre a massa de água contida no corpo de prova e a massa do corpo de prova seco, dado pela Equação 1:

$$
U(\%)=\frac{\mathrm{m}_{\mathrm{e}}-\mathrm{m}_{\mathrm{s}}}{\mathrm{m}_{\mathrm{s}}} \times 100,
$$

onde: $U(\%)$ - teor de umidade $(\%) ; m_{E}$ - massa do corpo de prova ao final do ensaio $(\mathrm{g}) ; m_{S}-$ massa seca do corpo de prova, após secagem (g).

\subsection{Determinação da resistência ao cisalhamento}

A resistência ao cisalhamento a uma dada temperatura é dada pela razão entre a força máxima atuante no corpo de prova e a área sujeita ao cisalhamento, como mostra a Equação 2:

$f_{v, T}=\frac{F_{v, m a ́ x}}{A_{v}}$,

onde: $f_{V, T}$ - resistência ao cisalhamento, em $(\mathrm{MPa})$, a uma dada temperatura $\mathrm{T} ; F_{V, M A ́ X}$ - força máxima aplicada na extremidade do corpo de prova, em $(\mathrm{N})$, a uma dada temperatura $\mathrm{T} ; A_{V}$ - projeção da área de reforço sobre o corpo de prova sujeita a cisalhamento, $\mathrm{em}\left(\mathrm{mm}^{2}\right)$.

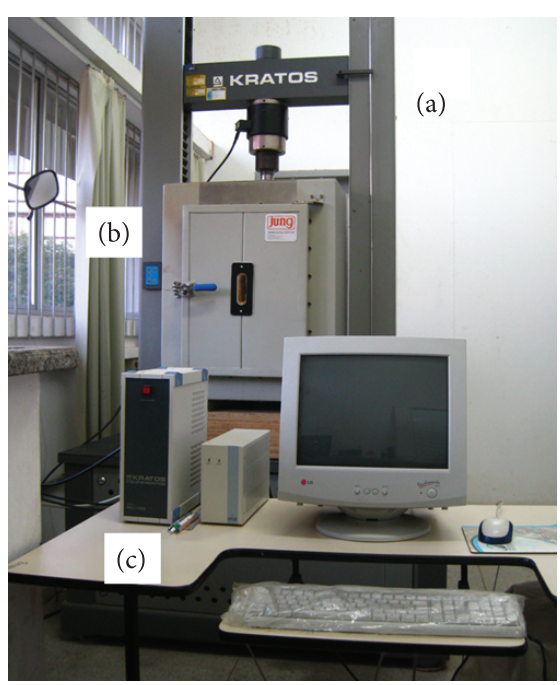

Figura 3. Dispositivo de ensaio: máquina universal de ensaios (a); câmara térmica (b); sistema de aquisição de dados (c).

Figure 3. Testing device: universal testing machine (a); thermal chamber (b); data acquisition system (c).

\subsection{Determinação da resistência ao cisalhamento relativa}

A resistência ao cisalhamento relativa foi obtida pela razão entre a resistência média ao cisalhamento, em uma dada temperatura, e a resistência média ao cisalhamento a $20^{\circ} \mathrm{C}$, e representada por $f_{V, T} / f_{V, 20^{\circ} \mathrm{C}}$.

\section{RESULTADOS E DISCUSSÃO}

Nesta seção, serão apresentados os resultados referentes ao teor de umidade, aos tipos de ruptura, à resistência de aderência do reforço à madeira e à análise da resistência ao cisalhamento relativa dos corpos de prova ensaiados.

\subsection{Teor de umidade}

A Figura 4 ilustra os resultados do teor de umidade dos corpos de prova após os ensaios mecânicos. Conforme esperado, o teor de umidade decresce linearmente com o aumento da temperatura, apresentando $8,8 \%$ de umidade a $80{ }^{\circ} \mathrm{C}$. Supõe-se que, no ensaio, foi eliminada somente a água livre existente nos corpos de prova. Segundo Young \& Clancy (2001), a libertação da umidade contida na parede celular pela superfície da madeira só ocorre a partir dos $100^{\circ} \mathrm{C}$.

\subsection{Tipos de ruptura dos corpos de prova}

Durante os ensaios, constataram-se dois tipos de falha: por cisalhamento na madeira e por ruptura na fibra, conforme indicado na Tabela 1, embora existisse a possibilidade de ocorrer o esmagamento na região do pino de fixação.

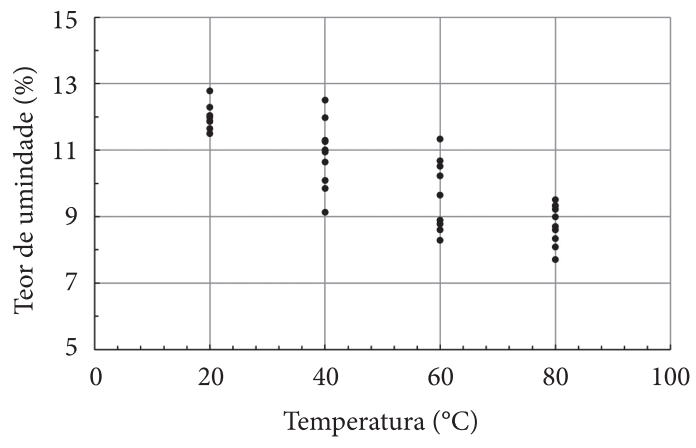

Figura 4. Teor de umidade dos corpos de prova reforçados.

Figure 4. Moisture content of the reinforced specimens. 
O padrão de ruptura dos corpos de prova pode ser verificado na Figura 5. Constata-se que esse padrão não se assemelha ao encontrado por Serra (2010), o qual ocorre com arrancamento de madeira. Tal situação pode indicar a necessidade de melhorar a aderência da superfície do corpo de prova, aumentando sua rugosidade antes da aplicação do reforço.

\subsection{Aderência do reforço à madeira}

A Figura 6 ilustra os resultados obtidos da resistência ao cisalhamento para o reforço de PRFC de 3,00 cm. Verifica-se que a resistência média e a dispersão dos resultados diminuem com o aumento da temperatura de ensaio.
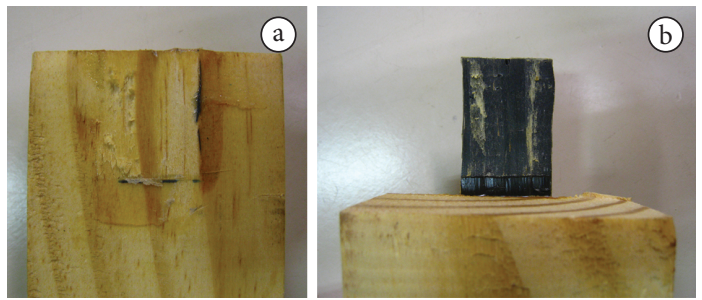

Figura 5. Padrão de ruptura observado nos corpos de prova.

Figure 5. Rupture pattern observed in the specimens.

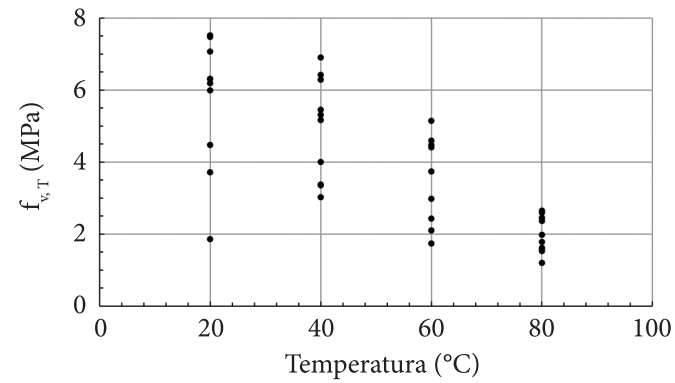

Figura 6. Resistência ao cisalhamento dos corpos de prova.

Figure 6. Shear strength of the specimens.
O tratamento estatístico realizado nos dados de ensaio mostra que o desvio padrão da aderência a $80^{\circ} \mathrm{C}$ é estatisticamente diferente dos desvios padrão encontrados nos outros níveis de temperatura. Dessa forma, a análise de variância foi realizada entre os dados de 20,40 e $60{ }^{\circ} \mathrm{C}$, indicando que, para a confiança de 95\%, há diferença estatisticamente significativa entre a aderência média a $20^{\circ} \mathrm{C}$ e aquela obtida a $60^{\circ} \mathrm{C}$.

A Figura 7 apresenta os resultados médios da aderência reforço-madeira obtidos nesta pesquisa e também aqueles obtidos por Serra (2010) em sua pesquisa sobre a resistência ao cisalhamento média do Pinus taeda, com o adesivo Sikadur ${ }^{\circledR}-330$ e com comprimento de aderência de $3,00 \mathrm{~cm}$. Observa-se que os resultados encontrados no presente trabalho foram, em média, 23,3\% inferiores aos obtidos por Serra (2010).

\subsection{Resistência ao cisalhamento relativa}

As médias de resistência relativas obtidas na presente pesquisa e naquela desenvolvida por Serra (2010) encontram-se representadas na Figura 8. Em

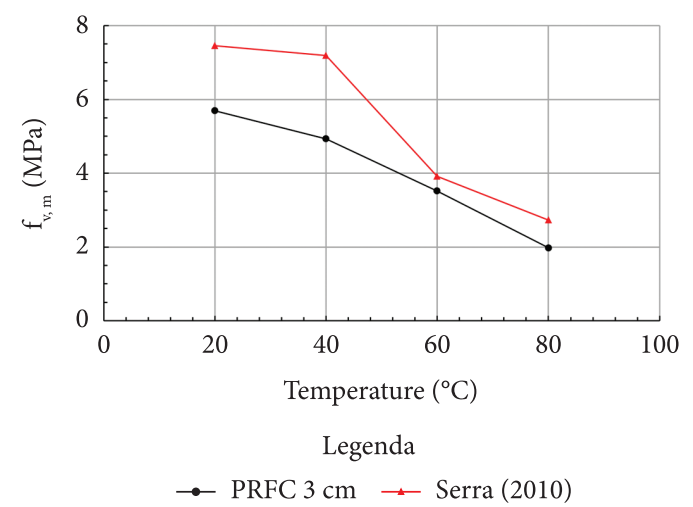

Figura 7. Comparação dos valores médios de resistência ao cisalhamento entre os trabalhos.

Figure 7. Comparison of mean values of shear strength between the works.

Tabela 1. Número de corpos de prova rejeitados na determinação da resistência ao corte.

Table 1. Number of rejected specimens in determining the shear strength.

\begin{tabular}{lcccc} 
Causa da rejeição & \multicolumn{4}{c}{ Número de corpos de prova rejeitados } \\
\cline { 2 - 5 } & $\mathbf{2 0}{ }^{\circ} \mathbf{C}$ & $\mathbf{4 0}{ }^{\circ} \mathbf{C}$ & $\mathbf{6 0}^{\circ} \mathbf{C}$ & $\mathbf{8 0}^{\circ} \mathbf{C}$ \\
\hline Esmagamento & 0 & 0 & 0 & 0 \\
Corte da fibra & 0 & 0 & 1 & 0 \\
Área de ruptura & 0 & 0 & 0 & 0 \\
Total & 0 & 0 & 1 & 0 \\
\hline
\end{tabular}




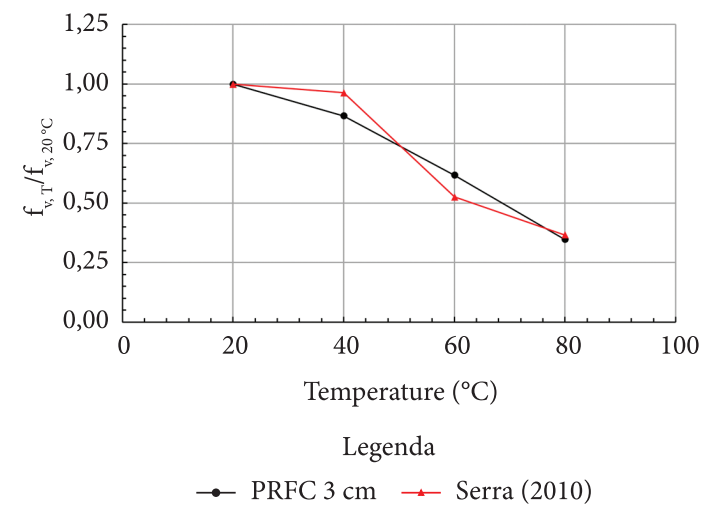

Figura 8. Comparação dos valores de resistência relativa.

Figure 8. Comparison of relative resistance values.

ambos os estudos, o adesivo utilizado foi do tipo resina epoxídica, porém de composição e fabricantes diferentes. Constatou-se que os resultados são aproximados e apresentam diminuição de resistência com o aumento da temperatura. A $60{ }^{\circ} \mathrm{C}$, a redução foi de $38,3 \%$, enquanto que, a $80{ }^{\circ} \mathrm{C}$, a redução foi de $65,3 \%$

\section{CONCLUSÕES}

Nesta pesquisa, foi estudada a influência das temperaturas entre 20 e $80{ }^{\circ} \mathrm{C}$ na resistência ao cisalhamento do reforço de fibra de carbono e resina epoxídica à madeira da espécie Pinus taeda, por meio de ensaios mecânicos de tração. Os resultados foram obtidos utilizando-se uma amostra de 40 corpos de prova de massa específica aparente média de $554 \mathrm{~kg} / \mathrm{m}^{3}$ e teor de umidade de $12,0 \%$ à temperatura ambiente, distribuídos em 4 níveis de temperatura, os quais foram pré-aquecidos por 120 minutos na temperatura de ensaio.

A partir dos resultados obtidos nesta pesquisa, chega-se a conclusão de que a resistência ao cisalhamento do reforço, especialmente na linha de cola, é afetada pelo aumento da temperatura, verificando-se a ruptura pela linha de cola em todos os níveis de temperatura de ensaio. Ainda, de acordo com os valores encontrados nos ensaios, conclui-se que:

- o teor de umidade dos corpos de prova diminui linearmente com o aumento da temperatura;
- a temperaturas superiores a $20^{\circ} \mathrm{C}$, houve uma redução de resistência uma vez que a linha de cola começou a ser influenciada pela temperatura; e

- a $60{ }^{\circ} \mathrm{C}$, a redução da resistência é de $38,3 \%$, enquanto que, a $80^{\circ} \mathrm{C}$, a resistência diminui $65,3 \%$ do valor à temperatura ambiente.

\section{AGRADECIMENTOS}

Os autores agradecem à Capes o suporte financeiro a esta pesquisa e ao $\mathrm{CNPq}$ a bolsa de iniciação científica do primeiro autor.

\section{STATUS DA SUBMISSÃO}

Recebido: 16/09/2011

Aceito: 08/02/2012

Resumo publicado online: 22/03/2012

Artigo completo publicado: 30/06/2012

\section{AUTOR(ES) PARA CORRESPONDÊNCIA}

\section{Lucas Rubini}

Grupo Interdisciplinar de Estudos da

Madeira - GIEM, Universidade Federal de Santa Catarina - UFSC, Campus Universitário Reitor João David Ferreira Lima, Trindade, CEP 88040-970, Florianópolis, SC, Brasil e-mail: lucasrubini@gmail.com

\section{Poliana Dias de Moraes}

Departamento de Engenharia Civil, Centro Tecnológico - CTC, Universidade Federal de Santa Catarina - UFSC, Rua João Duarte da Silva, 205, Córrego Grande, CEP 88037-000, Florianópolis, SC, Brasil e-mail: ecv1pdm@ecv.ufsc.br

\section{REFERÊNCIAS}

Associação brasileira de normas técnicas - ABNT. NBR 7190: Projeto de estruturas de madeira. Rio de Janeiro: ABNT; 1997.

Associação Brasileira de Normas Técnicas - ABNT. Projeto ce-02:126.10: Revisão: projeto de estruturas de madeira. Rio de Janeiro: ABNT; 2003. Anexo G: classificação visual de madeira serrada de coníferas destinada a aplicações estruturais. 
Balseiro A. Reforço e reabilitação de vigas de madeira por pré-esforço com laminados FRP [dissertação]. Porto: Faculdade de Engenharia, Universidade do Porto; 2007.

Bakis CE, Bank LC, Brown VL, Cosenza E, Davalos JF, Lesko JJ et al. Fiber-Reinforced Polymer Composites for construction - state-of-the-art review. Journal of Composites for Construction 2002; 6(2): 73-87. http:// dx.doi.org/10.1061/(ASCE)1090-0268(2002)6:2(73)

Fiorelli J, Dias AA. Utilização de fibras de carbono e de fibras de vidro para reforço de vigas de madeira [dissertação]. São Carlos: Escola de Engenharia de São Carlos, Universidade de São Paulo; 2002.

Frihart CR. Wood adhesion and adhesives. In: Rowell RM, editor. Handbook of Wood Chemistry and Wood Composites. Boca Raton: Taylor and Francis; 2005.

Garcez MR, Meneghetti LC, Cauduro LB, Campagnolo JL, Silva Filho LCP. Reforços de estruturas com polímeros à base de fibras. In: Anais do II Seminário de Patologia das Edificações; 2004; Porto Alegre. Porto Alegre: Universidade Federal do Rio Grande do Sul; 2004.

MC-Bauchemie. MC-DUR 1209: Resina de laminação para reforço estrutural com manta de fibra de carbono. [cited 2011 mai. 6]. Available from: http://www.mc- bauchemie.com.br/download.aspx?file=/datasheets $/$ technical/MC-DUR\%201209.pdf

Montgomery C, Runger C. Applied statistics and probability for engineers. 3rd ed. New York: Wiley; 2003.

Motavalli M, Czaderski C. FRP composites for retrofitting of existing civil structures in Europe: stateof-the-art review. In: Proceedings of the Composites of Polycon; 2007; Tampa. Tampa: American Composites Manufacturers Association - ACMA; 2007.

Nishizaki I, Takeda N, Ishizuka Y, Shimomura T. A case study of life cycle cost based on a real FRP bridge. In: Proceedings of the III International Conference on FRP Composites in Civil Engineering - CICE 2006; 2006; Miami. Miami; 2006.

Serra MCG. Estudo da aderência a altas temperaturas entre madeira e materiais de reforço [dissertação]. Coimbra: Faculdade de Ciências e Tecnologia, Coimbra; 2010.

Young S, Clancy P. Compression Mechanical Properties of Wood at Temperatures Simulating Fire Conditions. Fire and Materials 2001; 25: 83-93. http://dx.doi. org/10.1002/fam.759 